\title{
Advances on GRB as cosmological tools
}

\author{
G. Ghirlanda \\ INAF-Osservatorio Astronomico di Brera. Via E. Bianchi 46, I-23807 Merate (LC), Italy
}

\begin{abstract}
Several interesting correlations among Gamma Ray Bursts (GRB) prompt and afterglow properties have been found in the recent years. Some of these correlations have been proposed also to standardize GRB energetics to use them as standard candles in constraining the expansion history of the universe up to $z>6$. However, given the still unexplained nature of most of these correlations, only the less scattered correlations can be used for constraining the cosmological parameters. The updated $E_{\text {peak }}-E_{\gamma}$ correlation is presented. Caveats of alternative methods of standardizing GRB energetics are discussed.
\end{abstract}

Keywords: Gamma Ray Bursts, Afterglow, Prompt Emission

PACS: $98.70 . \mathrm{Rz}$, 95.85.Pw, 95.85.Nv, 95.30.Gv

\section{INTRODUCTION}

Different cosmological probes seem to point toward a consensus scenario characterized by a universe which is experiencing an accelerated expansion. Standard candles (SNIa, e.g. [1] ), standard rulers (clusters, CMB and BAO, e.g. [2]) and the angular power spectrum of the CMB (e.g. [3]) are used to probe the Hubble expansion flow up to redshifts 1-2 (by means of SNIa or clusters) or up to the epoch of recombination (through the CMB anisotropies).

Gamma Ray Bursts are observed up to very high redshifts: the farthest is GRB080913C at $z=6.7$ [4]. Among GRBs with known redshifts, 45\% are at $z>2$ and $8 \%$ at $z>4$. The high GRB luminosity $\left(10^{51} \mathrm{erg}\right)$ and their detection in the $\gamma$-ray band makes them attractive as a potential and complementary cosmological tool to constrain the cosmological models at $z>2$. However, the problem is that GRBs are all but standard candles [5]: their isotropic equivalent energetics and luminosities span 3-4 orders of magnitudes. Similarly to SNIa, it has been proposed to use correlations between various properties of the prompt emission [6, 7] and also of the afterglow emission [8, 9, 10, 11] to standardize GRB energetics.

\section{STANDARDIZING GRB ENERGETICS}

Isotropic energies (luminosities) can be computed for GRBs with measured redshifts and well constrained spectral properties. The spectrum gives the bolometric fluence $F$ (peak flux $P$ ) and then $E_{\text {iso }}=4 \pi d_{L}(z)^{2} F /(1+z)\left(L_{\text {iso }}=4 \pi d_{L}(z)^{2} P\right)$. These two quantities are strongly correlated with the rest frame peak energy $E_{\text {peak }}$ of the $v F_{v}$ spectrum [12, 13].

We have updated the sample of bursts with known $z$ and spectral parameters to Jan 2009 (the last being GRB 090102 at $\mathrm{z}=1.547$ ). These are 97 GRBs. The fit of both corre- 


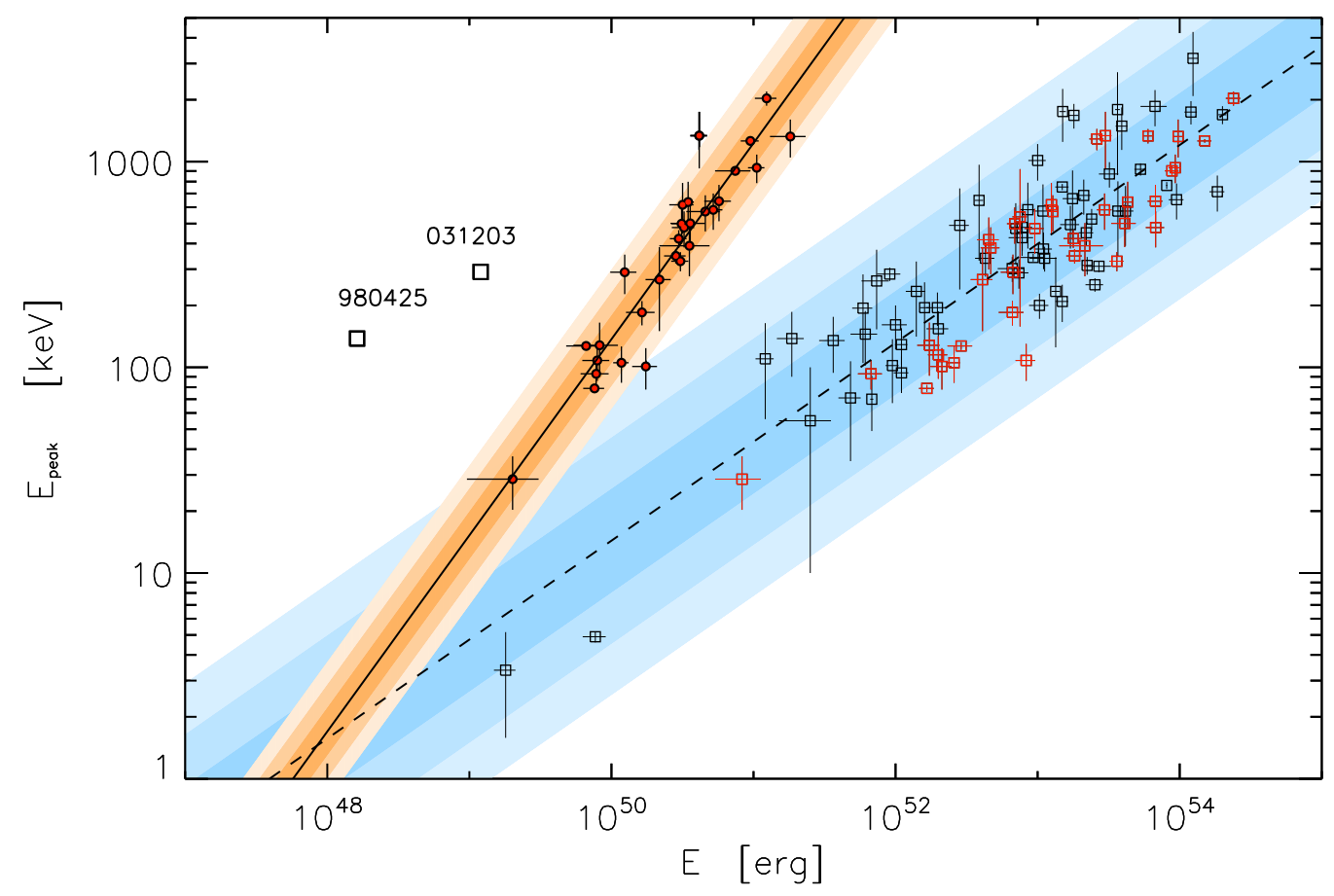

FIGURE 1. Correlation between the rest frame peak energy and the isotropic equivalent energy (open symbols - 97 GRBs) or the collimation corrected energy (filled symbols - 29 GRBs). The solid (dashed) line is the best fit of the $E_{\text {peak }}-E_{\gamma}\left(E_{\text {peak }}-E_{\text {iso }}\right)$ correlation. The shaded regions show the $1,2,3 \sigma$ dispersion (modeled as a Gaussian) of the data points around the best fit lines. Red open symbols are the GRBs with a jet break time measurement reported in the literature.

lations with a powerlaw gives $E_{\text {peak }} \propto E_{\text {iso }} 0.48 \pm 0.03$ and $E_{\text {peak }} \propto L_{\text {iso }} 0.4 \pm 0.03$. However, due to the large scatter of the data points $\chi^{2}$ is extremely large (492 and 612 for 95 degrees of freedom for the $E_{\text {peak }}-E_{\text {iso }}$ and $E_{\text {peak }}-L_{\text {iso }}$ correlations, respectively). The scatter of the data points is defined by their distance from the best fit line. By modeling the scatter distribution with a Gaussian we find a logarithmic dispersion of $\sigma=0.23$ dex and $\sigma=0.28$ dex for the $E_{\text {peak }}-E_{\text {iso }}$ and $E_{\text {peak }}-L_{\text {iso }}$ correlation, respectively. These scatters are much larger than the statistical errors associated with the observables $\left\langle\sigma_{E_{\text {peak }}}\right\rangle=0.1$, $\left\langle\sigma_{E_{\text {iso }}}\right\rangle=0.06,\left\langle\sigma_{L_{\text {iso }}}\right\rangle=0.07$. There is then the possibility that a third variable is responsible for this large scatter.

The large dispersion of the $E_{\text {peak }}-E_{\text {iso }}$ and $E_{\text {peak }}-L_{\text {iso }}$ correlations prevents their use to standardize GRB energetics. However, GRBs are thought to be collimated sources. In the standard GRB model the jetted outflow should produce a break in the afterglow light curve decay [14]. The measure of the time of occurrence of this break $t_{\text {jet }}$ allows to infer the jet opening angle $\theta_{\text {jet }}$ (under the standard afterglow model assumptions) and to recover the collimation corrected energy $E_{\gamma}=E_{\text {iso }}\left(1-\cos \theta_{\text {jet }}\right)$.

By collecting available estimates of jet break times from the literature we found [8] and later confirmed [15, 16] that the collimation corrected energy is strongly correlated 




FIGURE 2. Constraints on the cosmological parameters obtained with the $E_{\text {peak }}-E_{\gamma}$ correlation updated to Jan 2009 (29 bursts - solid line) compared to the previous update (19 GRBs - dashed line - [16]). Also shown are the constraints obtained from 156 SN type Ia (blue thin line - [17]) and those from the WMAP data (green thin line).

with $E_{\text {peak }}$. In Fig.1 the $E_{\text {peak }}-E_{\gamma}$ correlation is shown through the 29 bursts having a jet break in their optical afterglow light curves. We find $E_{\text {peak }}=E_{\gamma}^{1.04 \pm 0.08}$ with $\chi^{2}=37.8$ for 27 degrees of freedom assuming a wind-like profile of the circumburst medium. This correlation has a dispersion $\sigma=0.07$ which is consistent with the average statistical uncertainties on $E_{\text {peak }}$ and $E_{\gamma}$ and it is much smaller than the dispersion of the $E_{\text {peak }}-E_{\text {iso }}$ and $E_{\text {peak }}-L_{\text {iso }}$ correlations. This result also suggests that the dispersion of the $E_{\text {peak }}-E_{\text {iso }}$ correlation is due to the jet opening angle: by correcting $E_{\text {iso }}$ for $\theta_{\text {jet }}$ (for each burst), the scatter of the $E_{\text {peak }}-E_{\text {iso }}$ correlation is reduced.

Due to its tightness the $E_{\text {peak }}-E_{\gamma}$ correlation can be used to constrain the cosmological parameters [8, 16]. The $E_{\text {peak }}-E_{\gamma}$ correlation, shown in Fig.1, was found by assuming a cosmological model (i.e. $\Omega_{M}=0.3, \Omega_{\Lambda}=h=0.7$ ). It would be a circular argument to use this particular correlation to constrain the cosmological parameters. We originally solved this problem by properly accounting for the dependence of the correlation from the cosmological parameters [8] or by adopting a Bayesian fitting method [6, 16].

Fig, 2] shows the cosmological constraints obtained through the $E_{\text {peak }}-E_{\gamma}$ correlation 
with the most updated sample of 29 GRB. These are compared with the constraints obtained with the sample of 19 GRBs [16]. Also in this case we have applied the Bayesian method that overcomes the circularity problem.

\section{ISSUES}

The $E_{\text {peak }}-E_{\gamma}$ correlation is derived in the standard uniform jet scenario assuming a constant radiative efficiency and either a uniform [8] or a wind [15] circumburst density profile. [10] discovered a completely empirical correlation $E_{\mathrm{iso}} \propto E_{\text {peak }}^{2} t_{\text {jet }}^{-1}$ between the three observables which are combined in the $E_{\text {peak }}-E_{\gamma}$ correlation. Also the empirical correlation, due to its low scatter, can be used to derive constraints on the cosmological parameters [10].

The mostly debated issue of the spectral-energy correlations in general is that they are due to selection effects [18]. It has also been claimed that the slope and normalization of the $E_{\text {peak }}-E_{\text {iso }}$ correlation evolve with redshift [19] Selection effects can be studied in the observational plane corresponding to these correlations: the $E_{\text {peak,obs }}-F$ and the $E_{\text {peak,obs }}-P$ planes, where $F$ and $P$ are the bolometic fluence and peak flux. We have studied [20, 21] two instrumental selection effects: the minimum flux required to trigger a burst (trigger threshold) and the minimum flux to properly analyze its prompt emission spectrum (spectral threshold). The former has been claimed to bias the $E_{\text {peak }}-E_{\text {iso }}$ correlation [18]. The sample of 76 bursts (updated to Sept. 2007) with measured redshifts is composed by GRBs detected by different instruments. For this reason we modeled the trigger threshold and the spectral analysis threshold of the different detectors.

We can exclude that the $E_{\text {peak }}-E_{\text {iso }}$ correlation is biased by the trigger threshold. Also we can exclude that the spectral threshold is biasing the pre-Swift sample. Instead, the Swift GRB sample (27 events) is biased by the spectral threshold. This is also due to the limited spectral energy range of the BAT instrument on-board Swift which limits the measure of $E_{\text {peak,obs }}$ in the $15-150 \mathrm{keV}$ energy range. By considering sub-samples of bursts at different redshifts, we also exclude that the $E_{\text {peak }}-E_{\text {iso }}$ correlation slope or normalization change.

Recently [21] added GRBs without redshift in the $E_{\text {peak,obs }}-F$ and $E_{\text {peak,obs }}-P$ planes to compare the distribution of bursts with respect to the two selection effects. To this purpose a sample of 100 faint BATSE bursts, representative of a larger population of 1000 objects, was analyzed. By means of this complete, fluence-limited, GRB sample, it was found that the fainter BATSE bursts have smaller $E_{\text {peak,obs }}$ than those of bright events. As a consequence, the $E_{\text {peak,obs }}$ of these bursts is correlated with the fluence, though with a slope flatter than that defined by bursts with z. Selection effects, which are present, are not responsible for the existence of such a correlation. About six per cent of these bursts are surely outliers of the $E_{\text {peak }}-E_{\text {iso }}$ correlation, since they are inconsistent with it for any redshift. $E_{\text {peak,obs }}$ also correlates with the peak flux, with a slope similar to the $E_{\text {peak }}-L_{\text {iso }}$ correlation. In this case, there is only one sure outlier. The scatter of the $E_{\text {peak,obs }}-P$ correlation defined by the BATSE bursts of this sample is significantly smaller than the $E_{\text {peak,obs }}-F$ correlation of the same bursts, while for

the bursts with known redshift the $E_{\text {peak }}-E_{\text {iso }}$ correlation is tighter than the $E_{\text {peak }}-L_{\text {iso }}$ one. Once a very large number of bursts with $E_{\text {peak,obs }}$ and redshift will be available, we 
expect that the $E_{\text {peak }}-L_{\text {iso }}$ correlation will be similar to that currently found, whereas it is very likely that the $E_{\text {peak }}-E_{\text {iso }}$ correlation will become flatter and with a larger scatter.

One of the main drawback of the $E_{\text {peak }}-E_{\gamma}$ correlation for cosmological use is that it still has few points: the original sample of 15 events [8] has only doubled since 2004. With the launch of Swift [22] several jet breaks were expected to be measured, especially in the X-ray band. Jet breaks should be achromatic because they are produced by a geometric effect. All the jet breaks used to compute $E_{\gamma}$ in the pre-Swift era were, instead, obtained from the optical light curves. Swift revealed a complex X-ray afterglow light curve: the early afterglow is often characterized by a steep decay followed by a shallow phase lasting thousands of seconds [23]. A characteristic break time is that ending the Xray shallow phase. [24] showed that this time is not a jet break. A possible interpretation is that the shallow phase is produced by a long lasting central engine activity [25] as also supported by the presence of strong precursors, post-cursors, and X-ray flares in a sizable fraction of bursts. Often the X-ray and the optical afterglow light curves do not track one another, suggesting that they are two different emission components.

We selected a sample of 33 Gamma Ray Bursts (GRBs) detected by Swift, with known redshift and optical extinction at the host frame [26]. The de-absorbed and $\mathrm{K}$-corrected $\mathrm{X}$-ray and optical rest frame light curves are modelled as the sum of two components: emission from the forward shock due to the interaction of a fireball with the circumburst medium and an additional component, treated in a completely phenomenological way. The latter can be identified, among other possibilities, as "late prompt" emission produced by a long lived central engine with mechanisms similar to those responsible for the production of the "standard" early prompt radiation. We find a good agreement with the data, despite of their complexity and diversity. Our approach allows us to interpret the behaviour of the optical and X-ray afterglows in a coherent way, by a relatively simple scenario. Within this context it is possible to explain why sometimes no jet break is observed; why, even if a jet break is observed, it is often chromatic; why the steepening after the jet break time is often shallower than predicted.

\section{CAN WE USE THE $E_{\text {peak }}-E_{\text {iso }}$ OR $E_{\text {peak }}-L_{\text {iso }}$ CORRELATIONS FOR COSMOLOGY?}

The use of the $E_{\text {peak }}-E_{\gamma}$ correlation for cosmology requires to measure the redshift, the prompt emission $E_{\text {peak,obs }}$ and $t_{\text {jet }}$. The latter is the most critical observable: the jet break is typically observed at 1-2 days after the trigger and the light curve needs to be sampled at much later epochs in order to infer $t_{\text {jet }}$ when the afterglow can be very dim, also because Swift detects higher redshift bursts than before [27].

Therefore, it is interesting to explore if other correlations can be employed to standardize GRB energetics. Recent attempts [28, 29] tried to use the $E_{\text {peak }}-E_{\text {iso }}$ and $E_{\text {peak }}-L_{\text {iso }}$ correlations. In this cases, however, one has to take into account that these correlations are affected by a dispersion which is much larger than the statistical uncertainty on the data points.

The scatter of these correlations is due to three terms. One is the statistical uncertainty in the measurements of the parameters $\sigma_{\text {stat }}$. A second contribution to the scatter comes from systematic errors $\sigma_{\text {sys }}$ which could also have a physical origin but is difficult to 


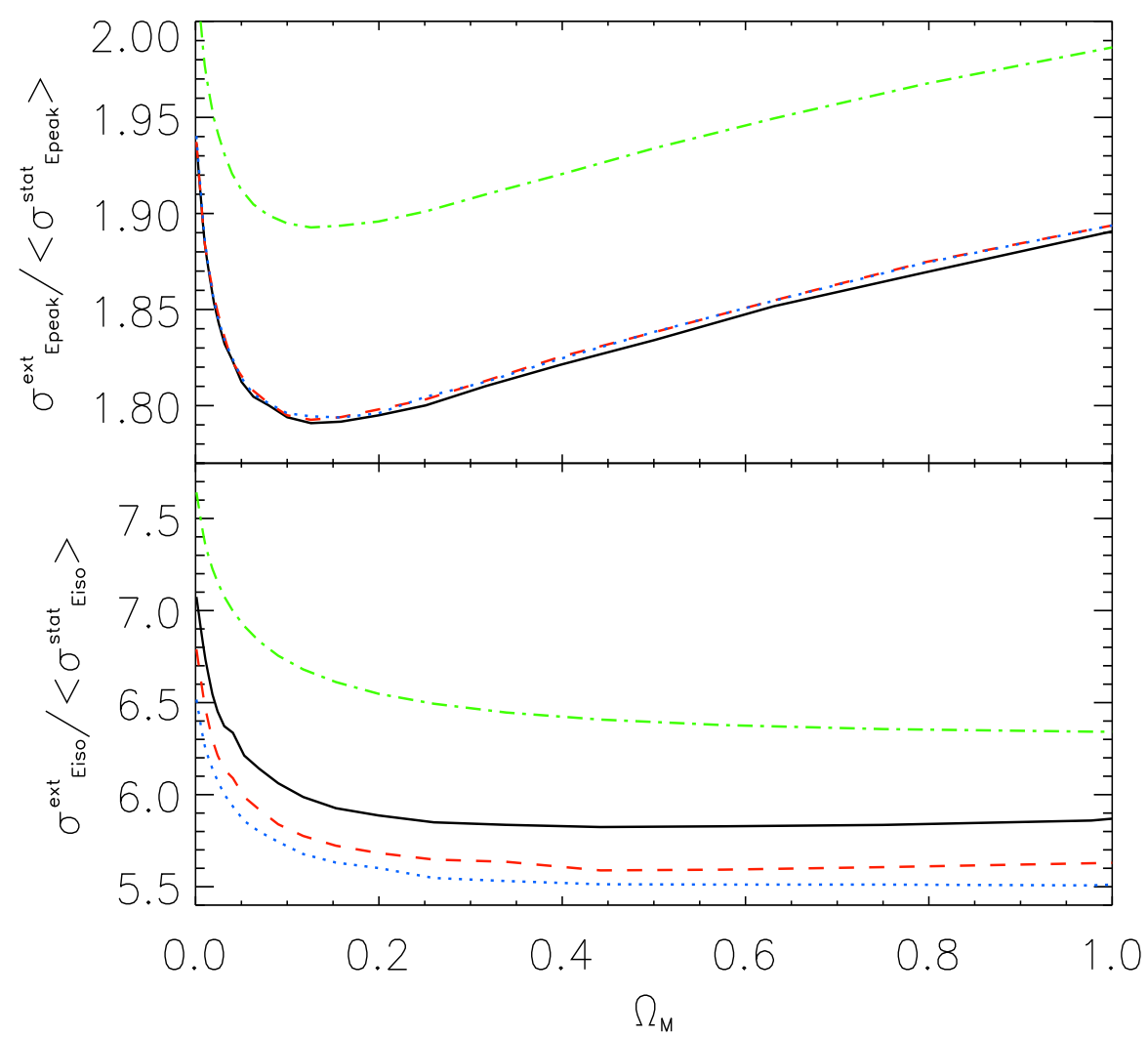

FIGURE 3. Test of the use of the $E_{\text {peak }}-E_{\text {iso }}$ correlation for constraining the cosmological parameters. The extra scatter terms of Eq.1, i.e. $\sigma_{x, y}$ vs $\Omega_{M}$ are normalized to the average value of the statistical error associate to the variable they are assigned to. Top panel: the extra-scatter term is assigned to $E_{\text {peak }}$ and the results similar to those of [28] are found. A minimum (though weak) is found for $\Omega_{M} \sim 0.15$. The solid curve is found with the likelihood function of Eq.1, the dotted and dashed curved are found with the symmetric likelihood function of [30]. The dot-dashed line is found with the $\chi^{2}$ fitting method. Bottom panel: the extra-scatter term is assigned to $E_{\text {iso. }}$ This is also the most obvious assumption as it is $E_{\text {iso }}$ to depend on the cosmological parameters. No minimum is found in this case of the extra-scatter term for any value of $\Omega_{M}$.

model. A third contribution to the scatter could finally be due to the cosmological model $\sigma_{\text {cosmo }}$ in the real cosmology its contribution should be minimized. In the $E_{\text {peak }}-E_{\text {iso }}$ and $E_{\text {peak }}-L_{\text {iso }}$ correlations the last two terms are dominating the scatter.

[28] ([29]) proposed the use of the $E_{\text {peak }}-E_{\text {iso }}\left(E_{\text {peak }}-L_{\text {iso }}\right)$ correlation to constrain the cosmological parameters. What is appealing is the possibility to use a correlation defined by a large GRB sample (much larger than that defining the $E_{\text {peak }}-E_{\gamma}$ correlation) and to use only on prompt emission observables ( $E_{\text {peak }}$ and $E_{\text {iso }}$ or $\left.L_{\text {iso }}\right)$.

However, in both correlations the non-statistical scatter $\sigma_{\text {sys }}^{2}+\sigma_{\text {cosmo }}^{2}$ need to be modeled. These terms (combined) are treated as a free parameter, i.e. the extra-scatter $\sigma_{\text {ext }}$ which is assumed to have a Gaussian distribution equal for all the data points.

To test the possibility of using the $E_{\text {peak }}-E_{\text {iso }}$ correlation for cosmology, [28] fit the $E_{\text {peak }}-E_{\text {iso }}$ correlation in different "cosmologies" (a flat Universe is assumed) and 
derive, in function of $\Omega_{M}$, the best fit values of the free parameters, i.e. the slope $m$ and the normalization $q$ of the correlation and the extra-scatter term $\sigma_{\text {ext }}$. The correlation is fitted with the likelihood function:

$$
\begin{array}{r}
\log P\left[m, q, \sigma_{x}, \sigma_{y} \mid\left(x_{i}, y_{i}, \sigma_{x, i}, \sigma_{y, i}\right)\right]=\frac{1}{2} \sum_{i} \log \left[\frac{1}{2 \pi\left(m^{2} \sigma_{x, i}^{2}+\sigma_{y, i}^{2}+m^{2} \sigma_{x}^{2}+\sigma_{y}^{2}\right.}\right]+ \\
-\frac{\left(y_{i}-m x_{i}-q\right)^{2}}{m^{2} \sigma_{x, i}^{2}+\sigma_{y, i}^{2}+m^{2} \sigma_{x}^{2}+\sigma_{y}^{2}}
\end{array}
$$

where $x_{i}, y_{i}, \sigma_{x, i}, \sigma_{y, i}$ are the data points with their statistical errors and $\sigma_{x}, \sigma_{y}$ are the projection of the extra-scatter $\sigma_{\text {ext }}$ along the coordinate axes.

[28] assume $\mathrm{Y}=E_{\text {peak }}, \mathrm{X}=E_{\text {iso }}$ and set the extra-scatter term $\sigma_{x}=0$, i.e. they give the (free) extra-scatter only to $E_{\text {peak }}$. They find that the extra-scatter $\sigma_{y}$ shows a minimum corresponding $\Omega_{M} \sim 0.1$. Therefore, they apply the standard procedure to derive constraints on the cosmological models through the $E_{\text {peak }}-E_{\text {iso }}$ correlation.

However, in the $E_{\text {peak }}-E_{\text {iso }}\left(E_{\text {peak }}-L_{\text {iso }}\right)$ correlations it is $E_{\text {iso }}\left(L_{\text {iso }}\right)$ that depends on the cosmological parameters (through the luminosity distance $d_{\mathrm{L}}\left(z \mid \Omega_{M}, \Omega_{\Lambda}, H_{0}\right)$ ). Therefore, the extra-scatter term should be assigned to $\mathrm{X}=E_{\text {iso }}$, i.e. $\sigma_{y}=0$.

For this reason we repeated the same test on the $E_{\text {peak }}-E_{\text {iso }}$ and $E_{\text {peak }}-L_{\text {iso }}$ correlations and we do not find any minimum of the extra-scatter $\sigma_{x}$ when it is assigned to $E_{\text {iso }}$ which is actually the variable that depends on the cosmological parameters. We verified our results also by (a) adopting the symmetric likelihood function of [30] (having the term $1+m^{2}$ in the numerator of the first term of Eq.1); (b) fitting with the least square method; (c) inverting the order of the fitting variables (i.e. setting $\mathrm{Y}=E_{\mathrm{iso}}$ and $\mathrm{X}=E_{\text {peak }}$ ). Similar results are also found for the $E_{\text {peak }}-L_{\text {iso }}$ correlation. Our results are shown in Fig. 3 ,

In the $E_{\text {peak }}-E_{\gamma}$ correlation (Fig.1) the scatter of the data points is already consistent with the statistical errors associated with $E_{\text {peak }}$ and $E_{\gamma}$. The only residual scatter is due to the cosmological model. This is why, without assumptions on the nature and "normality" of the unknown extra-scatter term, the $E_{\text {peak }}-E_{\gamma}$ correlation is preferable to standardize the GRB energetics. The $E_{\text {peak }}-E_{\gamma}$ correlation also proves that most of the scatter of the $E_{\text {peak }}-E_{\text {iso }}$ correlation is due to the jet opening angle, which is different from burst to burst.

This shows (as also recently demonstrated by [31]) that the $E_{\text {peak }}-E_{\text {iso }}$ and $E_{\text {peak }}-$ $L_{\text {iso }}$ correlations cannot be used straightforwardly to constrain the cosmological parameters due to the unknown nature of the extra-scatter they are affected by.

\section{ACKNOWLEDGMENTS}

I am grateful to D. Burlon, A. Celotti, C. Firmani, G. Ghisellini, M. Nardini, L. Nava, F. Tavecchio for collaborations and discussions. ASI is thanked for I/088/06/0 grant. 


\section{REFERENCES}

1. Astier, P., Guy, J., Regnault, N., et al., A\&A, 2006, 447, 31

2. Percival, W. J., Cole, S., Eisenstein, D. J., et al., MNRAS, 2007, 381, 1053

3. Lewis, A., $P h R v D, 2008,78,3002$

4. Greiner, J., Kruehler, T., Fynbo, J. P. U., et al., ApJ subm. (arXiv:0810.2314), 2009

5. Bloom, J. S., Frail, D. A., Kulkarni, S. R., ApJ, 2003, 549, 674

6. Firmani, C., Ghisellini, G., Avila-Reese, V., et al., MNRAS, 2006, 370, 185

7. Firmani, C., Avila-Reese, V., Ghisellini, G., et al.; MNRAS, 2006, 372, L28

8. Ghirlanda, G., Ghisellini, G., Lazzati, D., ApJ, 2004, 616, 331

9. Ghirlanda, G., Ghisellini, G., Lazzati, D., et al., ApJ, 2004, 613, L13

10. Liang, E. and Zhang, B., ApJ, 2005, 633, 611

11. Schaefer, B., ApJ, 2007, 660, 16

12. Amati, L., Frontera, F., Tavani, M., et al., $A \& A$, 2002, 390, 81

13. Yonetoku, D., Murakami, T., Nakamura, T., et al., ApJ, 2004, 609, 935

14. Rhoads, J., E., ApJ, 1997, 487, L1

15. Nava, L.; Ghisellini, G.; Ghirlanda, G., et al., $A \& A$, 2006, 450, 471

16. Ghirlanda, G.; Ghisellini, G.; Firmani, C., $N J P h, 8,123$

17. Riess, A. G., Strolger, L.-G., Tonry, J., et al., ApJ, 2004, 607, 665

18. Butler, N. R., Kocevski, D., Bloom, J. S., AIPC, 1000, 16

19. Li, Li-Xin, MNRAS, 2007, 379, L55

20. Ghirlanda, G., Nava, L., Ghisellini, G., et al., MNRAS, 2008, 387, 319

21. Nava, L., Ghirlanda, G., Ghisellini, G., et al., MNRAS, 2008, 391, 639

22. Gehrels, N., Chincarini, G., Giommi, P., et al., ApJ, 2004, 611, 1005

23. Burrows, D. N., Romano, P., Falcone, A., et al., Sci, 2005, 309, 1833

24. Nava, L., Ghisellini, G., Ghirlanda, G., et al., MNRAS, 2007, 377, 1464

25. Ghisellini, G., Ghirlanda, G., Nava, L., et al., ApJ, 2007, 658, L75

26. Ghisellini, G., Nardini, M., Ghirlanda, G., et al., MNRAS in press, arXiv0811.1038, 2009

27. Berger, E., Kulkarni, S. R., Fox, D. B., et al., ApJ, 2005, 634, 501

28. Amati, L., Guidorzi, C., Frontera, F., et al., MNRAS, 2008, 391, 577

29. Kodama, Y., Yonetoku, D., Murakami, T., et al., MNRAS, 2008, 391, L1

30. Reichart, D. E., ApJ, 2001, 553, 235

31. Basilakos, S. \& Perivolaropoulos, L., MNRAS, 2008, 391, 411 